\title{
Ciclosporin! A significant role in Resistant Dermatomyositis
}

\author{
Aravinthan. $N^{1}$, Vijeyaratnam. $D^{1}$ \\ ${ }^{1}$ Rheumatology Unit, Teaching Hospital Jaffna, Sri Lanka.
}

\section{Introduction}

Inflammatory myopathies represent largest group of acquired and potentially treatable cause of skeletal muscle weakness. The goal of therapy is to improve muscle strength and thereby improving function in activities of daily living and ameliorate extra muscularmanifestations. Though almost all patients respond to glucocorticoids, approximately $75 \%$ of patients ultimately require additional treatment with Azathioprine,Methotrexate or Mycophenolate (Mofetil).(1)In infrequent resistant cases evidence of clinically significant benefit is greatest with rituximab and intravenous Immunoglobulin. Here we report a case with resistant Dermatomyositis who failed to respond to Methotrexate, Mycophenolate (MMF), Azatioprine\& even no response to intravenous immunoglobulin, but well responded to Ciclosporin. $(1,4)$

\section{Case report}

A 55-year-old previously healthy lady until 2015 presented to the Rheumatology clinic with two week history of rash involving her face and hands and generalized body weakness. She initially noticed pain of proximal muscle of left sided arm with weakness followed up by pain and weakness of left lower limb mainly involved proximal muscle group and ultimately ended up in involvement of all four limbs which made her bedbound within 2 weeks of onset.

She never had any experience of numbness of hands or feet and there was no history of dysphgia, dysphonia or diplopia. She also noticed a non-itchy rash on her face and hands. She was not taking any regular medication until this event. She worked as a teacher.

Examination revealed a classical heliotrope rash as well as evidence of Gottron's papules and "V" sign of neck. She had bilateral upper and lower limb weakness in the proximal (1/5) and distal $(4 / 5)$ muscle groups as well as slight neck muscle weakness $(4 / 5)$. She was afebrile and all of her vital signs were normal.

Routine blood tests showed elevated ESR $(109 \mathrm{~mm})$,
CRP(50mg/dl),Creatine kinase (CK) (4000units/L) and LDH $(740 \mathrm{U} / \mathrm{L})$. Electromyography showed the features of myopathy and which was further confirmed by needle muscle biopsy as Dermatomyositis and the diagnosis was supported by positive ANA at 1:320 and positive anti jo anti body. The ANCA, Hepatitis B, C status, CT of chest, abdomen were normal. Biopsy of skin was consistence with Dermatomyositis.

She was commenced on prednisolone $1 \mathrm{mg} / \mathrm{kg}$ for which she responded well initially. However, with tapering of steroids she developed relapse evidenced by worsening of weakness and elevated level of routine CPK. The treatment with Methotrexate and later with the subsequent relapses Azathioprine and Mycophenolate were added.

During the follow up, in 2017 she relapsed, which was precipitated by osteomyelitis of tibial bone and she was treated for six weeks with IV and oral antibiotics. While on treatment for Osteomyelitis her usual DMARDS were withheld and flare up of Dermatomyositis was managed with methylprednisolone $500 \mathrm{mg}$ IV daily for 3 days and then with high dose of oral steroid $(1 \mathrm{mg} / \mathrm{kg})$. After completion of antibiotics for Osteomyeltis her usual DMARDS were recommenced gradually. However, she continued to have the features of flare up of Dematomyositis despite the three usual DMARDS and steroid.

Therefore this episode was regarded as a manifestation of resistant Dermatomyositis. Although multiple options are available for treating the refractory cases, evidence of clinically significant benefit is greatest with Rituximab \& IVimmunoglobulin. Even though Rituximab is usually considered as superior to Immunoglobulins, we had to treat with Immunoglobulin $2 \mathrm{mg} / \mathrm{kg}$, as she was recently treated for Osteomyelitis( the risk of flare up of infection with Rituximab) and unavailability of Rituximab at our hospital. However, even with Immunoglobulin she did have no improvement in her disease activity.

The diagnosis was reevaluated and reconfirmed with repeat muscle biopsy and EMG. Steroid 
induced myopathy was considered as one of the differential diagnosis and which was excluded by elevated CPK. Associated electrolyte imbalances and Endocrine abnormalities causing muscle weaknesses had been looked for and ruled out. She was screened for underlying malignancy causing refractory Dermatomyositis, earlier with noninvasive investigations such as CXR, HRCT chest, CT abdomen, mammography, Ultrasound of pelvis\&abdomen, blood picture and tumor markers such as CA-125, beta HCG and later with invasive upper gastrointestinal endoscopy, however all of which had revealed no evidence of malignancy.

As the final remedy she was started with a trial of calcineurin inhibitor, Ciclosporin $2.5 \mathrm{mg} / \mathrm{Kg} /$ day for which fortunately she did have dramatic response. Although she developed hirsutism with Ciclosporin, it was continude was taped, as tapering the drug made the tendency to develop relapse. Now with Ciclosporin she is doing well andleading a healthy life without recurrent flare up. We plan to do annual screening for malignancy as associated cancer may not become apparent for 2-3 years.

\section{Discussion}

Dermatomyositis is a rare disorder of unknown cause with the prevalence of 1 in 100000 . Its autoimmune a etiology is supported by associated other autoimmune, connective tissue diseases \& $\mathrm{T}$ cell mediated myocytotoxicity and complement mediated microangiopathy

Dermatomyositis can affect both children \& adults, Women are affected more often than men.It is a distinctive entity identified by characteristic rash accompanying or more often preceding muscle weakness.(2) Even though typical cases present as progressive symmetrical weakness, our patient initially developed unilateral weakness with asymmetrical distribution which favors Inclusion body myositis rather than Dermatomyositis, however initial muscle biopsy did reveal no features of inclusion bodies.Extramuscular manifestations may be present to a varying degree in patients with Dermatomyositis.(3)

The treatment of inflammatory myopathies to date has been complicated by the rarity of the disease and paucity of large randomized clinical trials. Sequential empirical treatment of Dermatomyositis is suggested as step 1 with high dose prednisolone, step 2 with Azathioprine,
Mycophenolate or Methotextrate for steroid sparing effect, step 3 with Intravenous immunoglobulins and step 4 with guarded optimism of one of the following agents chosen according to the patient's age, degree of disability, tolerance, experience with drugs and general health, so the drugs in step 4 are Rituximab,Ciclosporine, Cyclophosphamide\&Tacrolimus.

In previous case reports by Danial J Wallace \& Allen L suggested significant improvement with combination therapy with Methotrexate \& Chlorambucil, by Grover \& Peshin highlighted the importance of Rituximab in resistant cases of Dermatomyositis and by J.Albayda\&L. (4) Christopherstine gave importance of IV immunoglobulin and other novel agents in refractory cases. However, our patient showed dramatic improvement to Ciclosporin. Ciclosporin is a calcineurin inhibitor that inhibits production and release of IL-2 and IL-2 induced activation of T lymphocytes. Here we would like to emphasize the importance of Ciclosporin as a remedy in resistant cases of Dermatomyositis. (5)

\section{Abbreviations}

CRP- $\mathrm{C}$ reactive protein, ANA- anti nuclear anti body, ANCA- antineutrophil cytoplasmic antibody, DMARDS- disease modifying antirheumatic drugs, CXR- chest Xray

\section{References}

1. S.Moghadam-Kia,R.Aggarwal and C.Oddis "Treatment of inflammatory myopathy:emerging therapies and therapeutic targets" Expert review of clinical Immunology 2015;11(11):1265-1275

2. K.Grover\&R.Peshin "Use of Rituximab in Refractory Dermatomyositis":A Case Report," Open Journal of Rheumatology and Autoimmune Diseases, Vol.3 No.1,2013,pp.31-32

3. K.Naha,S.Karanth, Sowjanya Dasari\&M . Prabhu"polymyositis- like syndrome with rhabdomyolysis in association with brucellosis" Asian Pacific Journal of Tropical Medicine 755756 (2012)

4. Albayda\&L.Christopherstine "Novel approaches in the treatment of myositis and myopathies" Therapeutic advances in Musculoskeletal Disease.2012 Oct;4(5):369-377

5. D.Wallace,A.Metzger, and K.White "Combination immunosuppressive treatment of streroid resistant Dermatomyositis/Polymyositis," Arthritis and Rheumatism, Vol.28,No.5(May 1985) 